\title{
Batalin-Vilkovisky formality for Chern-Simons theory
}

\section{Ezra Getzler}

Department of Mathematics, Northwestern University, Evanston, Illinois 6065\%, U.S.A.

E-mail: getzler@northwestern.edu

ABSTRACT: We prove that the differential graded Lie algebra of functionals associated to the Chern-Simons theory of a semisimple Lie algebra is homotopy abelian. For a general field theory, we show that the variational complex in the Batalin-Vilkovisky formalism is a differential graded Lie algebra.

Keywords: Chern-Simons Theories, BRST Quantization, Topological Field Theories

ArXIV EPrint: 2110.10209 


\section{Contents}

1 Introduction 1

2 The Soloviev bracket and the Batalin-Vilkovisky classical master equation 4

3 Covariant field theories 12

4 The covariant abelian Chern-Simons action 15

5 The covariant non-abelian Chern-Simons action $\quad 19$

A The Chevalley-Eilenberg complex of a semisimple Lie algebra 20

\section{Introduction}

In the Batalin-Vilkovisky formalism, functionals form a sheaf of graded Lie superalgebras over spacetime, with differential given by the inner derivation with respect to the classical action:

$$
\mathrm{s} \int f=\left(\int S, \int f\right) .
$$

The functionals are graded by the ghost number, and the antibracket $\left(\int f, \int g\right)$ has degree 1 . In particular, cohomology classes of degree -1 form a Lie superalgebra, which generate the symmetries of the theory, cohomology classes of degree 0 represent infinitesimal deformations of the action $\int S$, and cohomology classes of degree 1 represent obstructions. The derivation $\mathrm{s}$ is a differential because $\int S$ satisfies the Batalin-Vilkovisky classical master equation

$$
\left(\int S, \int S\right)=0
$$

Following Sullivan [12] and Goldman and Millson [8], it is natural to study whether these differential graded $(\mathrm{dg})$ Lie superalgebras are formal. A formal dg Lie superalgebra $L$ is a dg Lie superalgebra that is weakly equivalent to its cohomology $H^{*}(L)$. In other words, there is a diagram of dg Lie superalgebras

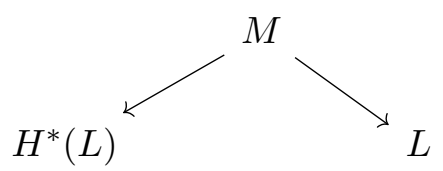

in which the arrows induce isomorphisms on cohomology. In particular, if there is a morphism of dg Lie superalgebras $\left(H^{*}(L), 0\right) \rightarrow(L, d)$ inducing an isomorphism on cohomology, then $L$ is formal. This is the only situation that will arise in this article. 
Formality is met in the deformation theory of compact Calabi-Yau manifolds: the $\mathrm{dg}$ Lie algebra $\left(\Omega^{0, *}(M, \Lambda T), \bar{\partial}\right)$, namely, the Dolbeault resolution of the Schouten algebra, is formal. By the Tian-Todorov theorem, more is true: the bracket induced on the cohomology $H^{*}(M, \Lambda T)$ by the Schouten bracket vanishes, so this dg Lie algebra is in fact weakly equivalent to an abelian graded Lie algebra. We say that $\Omega^{0, *}(M, \Lambda T)$ is homotopy abelian.

In this article, we show that a similar property holds for the Batalin-Vilkovisky cohomology for the Chern-Simons theory associated to a semisimple Lie algebra $\mathfrak{g}$. ChernSimons theory is a classical field theory on $\mathbb{R}^{3}$ associated to a real Lie algebra $\mathfrak{g}$ and an invariant non-degenerate symmetric bilinear form $\langle x, y\rangle$. Let $A=A_{1} d t^{1}+A_{2} d t^{2}+A_{3} d t^{3}$ be a connection one-form on $\mathbb{R}^{3}$, with values in the Lie algebra $\mathfrak{g}$. The Chern-Simons theory has action

$$
\int \operatorname{cs}_{3}(A),
$$

where $\operatorname{cs}_{3}(A)$ is the Chern-Simons 3 -form

$$
\operatorname{cs}_{3}(A)=d t^{i} d t^{j} d t^{k}\left(\frac{1}{2}\left\langle A_{i}, \partial_{j} A_{k}\right\rangle+\frac{1}{6}\left\langle A_{i},\left[A_{j}, A_{k}\right]\right\rangle\right) .
$$

The Euler-Lagrange equation is

$$
\frac{\delta \mathrm{cs}_{3}(A)}{\delta A_{i}}=\frac{1}{2} \epsilon^{i j k} F_{j k}=0,
$$

where $F_{i j}=\partial_{i} A_{j}-\partial_{j} A_{i}+\left[A_{i}, A_{j}\right]$, and the classical solutions of the theory are precisely the flat connections.

In addition to the gauge field $A$, Batalin and Vilkovisky consider a fermionic ghost field $c$, also with values in $\mathfrak{g}$, of ghost number 1 , and antifields $A^{+i}$ and $c^{+}$, of ghost number -1 and -2 respectively. Let $\epsilon_{i j k}$ and $\epsilon^{i j k}$ be the antisymmetric tensors with $\epsilon_{123}=1$ and $\epsilon^{123}=1$ : these tensors correspond to a choice of orientation of $\mathbb{R}^{3}$. The fields $A$ and $c$ and their antifields $A^{+}$and $c^{+}$are the components of a superconnection form

$$
\mathbb{A}=c+d t^{i} A_{i}+\frac{1}{2} \epsilon_{i j k} d t^{i} d t^{j} A^{+k}+d t^{1} d t^{2} d t^{3} c^{+} .
$$

Let $\operatorname{cs}_{3}(\mathbb{A})$ be the Chern-Simons 3-form, the 3-form component of the differential form

$$
\frac{1}{2}\langle\mathbb{A}, d \mathbb{A}\rangle+\frac{1}{6}\langle\mathbb{A},[\mathbb{A}, \mathbb{A}]\rangle .
$$

Axelrod and Singer [1] identified this form as the solution of the Batalin-Vilkovisky classical master equation for Chern-Simons theory:

$$
\frac{1}{2}\left(\left(\int \operatorname{cs}_{3}(\mathbb{A}), \int \operatorname{cs}_{3}(\mathbb{A})\right)\right)=0 .
$$

Let $\mathcal{A}$ be the algebra of differential polynomials in the coordinates $\left\{t^{1}, t^{1}, t^{3}\right\}$ of $\mathbb{R}^{3}$ and the fields $\left\{c, A_{i}, A^{+i}, c^{+}\right\}$, or the polynomial algebra generated by the coordinates on the jet space

$$
\left\{t^{i}, \partial^{\alpha} c, \partial^{\alpha} A_{i}, \partial^{\alpha} A^{+i}, \partial^{\alpha} c^{+} \mid i \in\{1,2,3\}, \alpha \in \mathbb{N}^{3}\right\} .
$$

This is a module for the Lie algebra of constant coefficient vector fields spanned by $\left\{\partial_{1}, \partial_{2}, \partial_{3}\right\}$, and we may form the variational complex

$$
0 \rightarrow \mathbb{R} \rightarrow \Omega^{0} \otimes \mathcal{A} \stackrel{d}{\rightarrow} \Omega^{1} \otimes_{\mathcal{O}} \mathcal{A} \stackrel{d}{\rightarrow} \Omega^{2} \otimes_{\mathcal{A}} \mathcal{A} \stackrel{d}{\rightarrow} \Omega^{3} \otimes_{\mathcal{A}} \mathcal{A} \stackrel{\int}{\rightarrow} \mathcal{F} \rightarrow 0 .
$$


Here, $\mathcal{O}$ is the sheaf of functions on $\mathbb{R}^{3}, \Omega^{k}$ is the sheaf of $k$-forms on $\mathbb{R}^{3}$ and $d$ is the de Rham differential

$$
d=\sum_{i=1}^{3} d t^{i} \partial_{i}
$$

The space $\mathcal{F}$ of functionals is the cokernel of the de Rham differential $d: \Omega^{2} \otimes_{\mathcal{O}} \mathcal{A} \rightarrow \Omega^{3} \otimes_{\mathcal{O}} \mathcal{A}$, and $\int$ is the projection from $\Omega^{3} \otimes_{\mathcal{O}} \mathcal{A}$ to $\mathcal{F}$. An important result in variational calculus, due to Vinogradov and Takens, is that the variational complex is exact (Olver [10, Theorem 5.56]).

Let $\mathbb{F}$ be the truncation of this sequence obtained by deleting the rightmost term $\mathcal{F}$ :

$$
0 \rightarrow \mathbb{R} \rightarrow \Omega^{0} \otimes_{\mathcal{O}} \mathcal{A} \stackrel{d}{\rightarrow} \Omega^{1} \otimes_{\mathcal{O}} \mathcal{A} \stackrel{d}{\rightarrow} \Omega^{2} \otimes_{\mathcal{O}} \mathcal{A} \stackrel{d}{\rightarrow} \Omega^{3} \otimes_{\mathcal{O}} \mathcal{A} \rightarrow 0
$$

Placing $\Omega^{k} \otimes_{\mathcal{O}} \mathcal{A}^{\ell}$ in degree $k+\ell-3$, we obtain a resolution of $\mathcal{F}$. In section 2 , we show that the Batalin-Vilkovisky antibracket on $\mathcal{F}$ lifts to an antibracket $((-,-))$ on $\mathbb{F}$, which we call the Soloviev antibracket, making $\mathbb{F}$ into a differential graded Lie algebra. This generalizes the case of a single independent variable $[6,7]$. In this general setting, we also show that a solution $\int S$ of the Batalin-Vilkovisky classical master equation lifts to a Maurer-Cartan element of $\mathbb{F}$, that is, a solution of the equation

$$
d S+\frac{1}{2}((S, S))=0 .
$$

This implies that the differential graded Lie algebra $\mathcal{F}$, with differential s given by the adjoint action of $\int S$, is resolved by the differential graded Lie algebra $\mathbb{F}$ with differential $d+\mathrm{s}$, where $\mathbf{s}=((S,-))$ is given by the adjoint action of $S$. The theory that we present is more general than is needed for our discussion of Chern-Simons theory, but we hope that the general case will find application in future work.

We also show that the differential $d+\mathrm{s}$ is equivalent to the differential $d+\mathrm{X}_{S}$ introduced by Gelfand and Dikii, where $\mathrm{X}_{S}$ is the Hamiltonian vector field associated to $S$. The differential $d+\mathrm{X}_{S}$ has the advantage that it is the total derivative of a double complex. This means that it is considerably easier to calculate its cohomology than to calculate directly the cohomology of $d+\mathrm{s}$. On the other hand, $d+\mathrm{X}_{S}$ is not a derivation of the Soloviev antibracket, meaning it has a less transparent relationship to the differential graded Lie algebra $\mathcal{F}$.

In section 4, following Barnich, Brandt and Henneaux [2], we calculate the cohomology of the differential $d+\mathrm{X}_{\Pi}$, where $\Pi$ is the action of the abelian Chern-Simons theory. From this, we obtain a quasi-isomorphism from the Chevalley-Eilenberg complex $\tau_{\geq 1} C^{*+3}(\mathfrak{g})$ (with vanishing differential) to $\mathbb{F}$ with differential $d+\pi$, where $\pi$ is the differential associated to the abelian Chern-Simons action $\Pi$. This remains a quasi-isomorphism of differential graded Lie algebras after passage to the non-abelian Chern-Simons theory. The differential on $\tau_{\geq 1} C^{*}(\mathfrak{g})$ is now the Chevalley-Eilenberg differential, allowing us to identify the cohomology of $\mathcal{F}$ with $\tau_{\geq 1} H^{*}(\mathfrak{g})$. In particular, if $\mathfrak{g}$ is semisimple, Whitehead's lemma implies that the cohomology $H^{*}(\mathcal{F}, \mathrm{s})$ vanishes in negative degrees, since $H^{1}(\mathfrak{g})=H^{2}(\mathfrak{g})=0$.

In summary, we have a diagram of three differential graded Lie algebras

$$
\tau_{\geq 1} C^{*+3}(\mathfrak{g}) \rightarrow \mathbb{F}^{*} \rightarrow \mathcal{F}^{*}
$$


where both arrows are quasi-isomorphisms. When $\mathfrak{g}$ is reductive, there is a quasi-isomorphism

$$
\tau_{\geq 1} H^{*}(\mathfrak{g}) \cong \tau_{\geq 1} C^{*}(\mathfrak{g})^{\mathfrak{g}} \hookrightarrow \tau_{\geq 1} C^{*}(\mathfrak{g})
$$

In the appendix, we show that the bracket induced on $\tau_{\geq 1} C^{*}(\mathfrak{g})^{\mathfrak{g}}$ by the bracket on $C^{*}(\mathfrak{g})^{\mathfrak{g}}$ vanishes if $\mathfrak{g}$ is semisimple. This is proved using Chevalley's theorem, that the algebra $I(\mathfrak{g})$ of invariant polynomials is spanned by trace polynomials $\operatorname{Tr}_{V}\left(\rho(x)^{k}\right)$, where $\rho: \mathfrak{g} \rightarrow \operatorname{End}(V)$ ranges over finite-dimensional representations of $\mathfrak{g}$ and $k$ over positive integers. This shows that the sheaf of differential graded Lie algebras $(\mathcal{F}, \mathrm{s})$ is homotopy abelian.

The explicit construction of the morphism of complexes $\tau_{\geq 1} C^{*+3}(\mathfrak{g}) \rightarrow \mathbb{F}^{*}$, and the proof that its composition with $\int: \mathbb{F} \rightarrow \mathcal{F}$ is a morphism of differential graded Lie algebras, is the heart of the paper. This may also be viewed as a lift of Proposition 2.5 of Cattaneo and Felder [4], which is a statement about general AKSZ models, from calculus to variational calculus.

To do this, we refine the classical master equation to incorporate equivariance with respect to the Lie algebra of constant vector fields on $\mathbb{R}^{3}$. Just as the Batalin-Vilkovisky classical master equation is the Maurer-Cartan equation for a differential graded Lie algebra, this refinement is the Maurer-Cartan equation for a curved differential graded Lie algebra structure on the space of local functionals tensored with the algebra of polynomials in variables $\left(u_{1}, u_{2}, u_{3}\right)$ of degree 2 . We call this the covariant extension of the classical master equation. Consider the differential polynomials

$$
G_{i}=\frac{1}{2} \epsilon_{i j k}\left\langle A^{+j}, A^{+k}\right\rangle+\left\langle A_{i}, c^{+}\right\rangle, \quad i \in\{1,2,3\} .
$$

Our main result is that the morphism that takes a function $f(c)$ of the field $c$, that is, an element $f \in C^{*}(\mathfrak{g})$, to the functional

$$
\left(\int G_{1},\left(\int G_{2},\left(\int G_{3}, \int f(c)\right)\right)\right) \in \mathcal{F}^{*-3},
$$

is a quasi-isomorphism of differential graded Lie algebras. We are even able to construct a lift of this morphism to $\mathbb{F}$, and show that this is also a morphism of differential graded Lie algebras, although in the proof that $(\mathcal{F}, \mathrm{s})$ is homotopy abelian, we do not really need that this lifted morphism preserves Lie brackets. However, this refinement may be of use in future work, especially in the study of boundary conditions.

\section{The Soloviev bracket and the Batalin-Vilkovisky classical master equation}

Let $M$ be a graded supermanifold, with coordinates $x^{a}$ of ghost number $\operatorname{gh}\left(x^{a}\right) \in \mathbb{Z}$ and parity $\mathrm{p}\left(x^{a}\right) \in \mathbb{Z} / 2$. The Batalin-Vilkovisky supermanifold $T^{*}[-1] M$ has coordinates $x^{a}$ and the dual coordinates (known in physics as antifields) $\xi_{a}$, with ghost number $\operatorname{gh}\left(\xi_{a}\right)=$ $-1-\operatorname{gh}\left(x^{a}\right)$ and parity $\mathrm{p}\left(\xi_{a}\right)=1-\mathrm{p}\left(x^{a}\right)$. A graded supermanifold carries an action of the Lie group GL(1), in other words, for each nonzero constant $a$, a dilation by $a$. The fixed-point set is a supermanifold in the usual sense, and by the body of the graded 
supermanifold, we mean the body of this fixed-point set. The body of $M$ has as coordinates the degree 0 coordinates of even parity of $M$. A sheaf on $M$ is a sheaf on its body.

Graded supermanifolds generalize graded manifolds, where the ghost number (degree) determines the parity. The introduction of a bigrading allows for fermionic physical fields. In Chern-Simons field theory, such fields are absent, and the parity of a field is the same as the reduction of its ghost number modulo 2 .

Fix a natural number $n$, the number of independent coordinates, and let $\mathcal{O}$ be the structure sheaf of $\mathbb{R}^{n}$. Consider the product $\mathbb{R}^{n} \times T^{*}[-1] M$, whose elements are functions in the coordinates $\left\{t^{i}, x^{a}, \xi_{a}\right\}$. The coordinates $t^{i}$ are the independent variables, and the coordinates $x^{a}$ and $\xi_{a}$ are referred to respectively as fields and antifields, or simply as fields.

Introduce jet coordinates $\left\{x_{\alpha}^{a}, \xi_{a, \alpha}\right\}$, where $\alpha$ ranges over positive $n$-dimensional multiindices:

$$
\alpha \in\left\{\left(\alpha_{1}, \ldots, \alpha_{n}\right) \in \mathbb{N}^{n} \mid \alpha>0\right\} .
$$

Let $|\alpha|=\alpha_{1}+\cdots+\alpha_{n}$; the notation $\alpha>0$ indicates that $|\alpha|>0$. Let $\mathcal{A}$ be the sheaf of graded commutative algebras on $U \times T^{*}[-1] M$ that are functions of the coordinates $\left\{t^{i}, \partial^{\alpha} x^{a}, \partial^{\alpha} \xi_{a} \mid 1 \leq i \leq 3, \alpha \geq 0\right\}$. (Different choices of function algebras may be made here: in practice, we choose functions that are (graded) polynomials in the coordinates of positive degree, power series in the coordinates of negative degree, and polynomial, real analytic, or infinitely differentiable in the coordinates of zero degree and the independent variables.) This is the sheaf of functions on the jet space $J_{\infty}\left(\mathbb{R}^{n}, M\right)$ of maps from $\mathbb{R}^{n}$ to $M$; we may think of the jet coordinates as the Taylor coefficients of a formal map from $\mathbb{R}^{n}$ to $M$ :

$$
x^{a}(t)=\sum_{\alpha} \frac{t^{\alpha} x_{\alpha}^{a}}{\alpha !}, \quad \xi_{a}(t)=\sum_{\alpha} \frac{t^{\alpha} \xi_{a, \alpha}}{\alpha !} .
$$

The space of vertical vector fields on the jet space is a free module over $\mathcal{A}$; denote the basis of vertical vector fields given by partial derivatives with respect to the jet coordinates by

$$
\partial_{\alpha, a}=\frac{\partial}{\partial x_{\alpha}^{a}}, \quad \partial_{\alpha}^{a}=\frac{\partial}{\partial \xi_{a, \alpha}} .
$$

Introduce the vector fields corresponding to infinitesimal translation in the coordinate $t^{i}$ :

$$
\partial_{i}=\frac{\partial}{\partial t^{i}}+\sum_{\alpha}\left(x_{\alpha+i}^{a} \partial_{a, \alpha}+\xi_{a, \alpha+i} \partial_{\alpha}^{a}\right) .
$$

Here, $\alpha+i$ is an abbreviation for the multi-index $\left(\alpha_{1}, \ldots, \alpha_{i}+1, \ldots, \alpha_{n}\right)$. A vector field $\tau$ is evolutionary if it commutes with the vector fields $\partial_{i}$. Such a vector field is determined by its action on the coordinates $\left\{t^{i}, x^{a}, \xi_{a}\right\}$ : it is given by the explicit formula

$$
\tau=f^{i} \partial_{i}+\operatorname{pr}\left(g^{a} \partial_{a}+h_{a} \partial^{a}\right)=f^{i} \partial_{i}+\sum_{\alpha}\left(\partial^{\alpha} g^{a} \partial_{a, \alpha}+\partial^{\alpha} h_{a} \partial_{\alpha}^{a}\right)
$$

where $f^{i}=\tau\left(t^{i}\right), g^{a}=\tau\left(x^{a}\right), h_{a}=\tau\left(\xi_{a}\right)$, and the prolongation is the vector field

$$
\operatorname{pr}\left(g^{a} \partial_{a}+h_{a} \partial^{a}\right)=\sum_{\alpha}\left(\partial^{\alpha} g^{a} \partial_{a, \alpha}+\partial^{\alpha} h_{a} \partial_{\alpha}^{a}\right) .
$$


The variational derivatives are the operators on $\mathcal{A}$ given by the formulas

$$
\delta_{a}=\sum_{\alpha \in \mathbb{N}^{n}}(-\partial)^{\alpha} \partial_{\alpha, a} \quad \delta^{a}=\sum_{\alpha \in \mathbb{N}^{n}}(-\partial)^{\alpha} \partial_{\alpha}^{a}
$$

These operators are not derivations, but rather differential operators of infinite order.

Let $\mathbb{F}^{*, *}$ be the bicomplex of sheaves

$$
\mathbb{F}^{-p, q}= \begin{cases}\Lambda^{p} \mathbb{R}^{n} \otimes \mathcal{A}^{q}, & 0 \leq p \leq n, \\ \mathbb{R} \eta, & p=n+1 \text { and } q=0 .\end{cases}
$$

If $I=\left\{i_{1}<\cdots<i_{p}\right\} \subset\{1, \ldots, n\}$, let $\sigma_{I}=\sigma_{i_{1}} \ldots \sigma_{i_{p}}$. If $p \leq n$, a typical element of $\mathbb{F}^{-p, q}$ is a sum

$$
f=\sum_{I=\left\{i_{1}<\ldots i_{p}\right\}} \sigma_{I} f_{I}
$$

where $\operatorname{gh}\left(f_{I}\right)=q$. The total degree of $f$ is $q-p$. The differential $d: \mathbb{F}^{-p, q} \rightarrow \mathbb{F}^{-p+1, q}$ equals

$$
d\left(\sigma_{I} f_{I}\right)=\sum_{\ell=1}^{k}(-1)^{\ell-1} \sigma_{I \backslash i_{\ell}} \partial_{i_{\ell}} f_{I}
$$

if $p \leq n$, while the differential from $\mathbb{F}^{-n-1, q} \rightarrow \mathbb{F}^{-n, q}$ takes $\eta$ to $\sigma_{1} \ldots \sigma_{n}$. The element $\sigma_{i_{1}} \ldots \sigma_{i_{k}} f_{I}$ of $\mathbb{F}$ corresponds to the differential form

$$
\left.\left.\left(\partial_{i_{1}}\right\lrcorner \ldots \partial_{i_{k}}\right\lrcorner d t^{1} \ldots d t^{d}\right) f_{I} .
$$

We will tacitly assume this identification of elements of $\mathbb{F}$ with differential forms.

We also consider the subspace $\mathbb{F}_{\circ} \subset \mathbb{F}$ made up of functions having no explicit dependence on the coordinates $\left\{t^{i}\right\}$, and the subalgebra $\mathcal{A}_{\circ}=\mathcal{A} \cap \mathbb{F}_{\circ} \subset \mathcal{A}$. On this subcomplex, the evolutionary vector field $\partial_{i}$ restricts to the vector field

$$
\operatorname{pr}\left(x_{i}^{a} \partial_{a}+\xi_{a, i} \partial^{a}\right)
$$

The differential $d: \mathbb{F}^{-p, *} \rightarrow \mathbb{F}^{-p+1, *}$ induces a differential on the subspace $\mathbb{F}_{\circ}$.

The cohomology

$$
\mathcal{F}=\mathbb{F}^{0, *} / d \mathbb{F}^{-1, *} \cong \mathcal{A} / \sum_{i=1}^{n} \partial_{i} \mathcal{A}
$$

of the differential $d$ in $\mathbb{F}^{0, *}$ is the sheaf of functionals of the classical field theory. The projection from $\mathbb{F}^{0, *} \cong \mathcal{A}$ to $\mathcal{F}$ is denoted by $f \mapsto \int f$ : in terms of differential forms, it corresponds to integration over differential forms of top degree. We also consider the cohomology

$$
\mathcal{F}_{\circ}=\mathbb{F}_{\circ}^{0, *} / d \mathbb{F}_{\circ}^{-1, *} \cong \mathcal{A}_{\circ} / \sum_{i=1}^{n} \partial_{i} \mathcal{A}_{\circ}
$$

of the differential $d$ in $\mathbb{F}_{o}^{0, *}$, consisting of functionals with no explicit dependence on the independent variables $\left\{t^{i}\right\}$.

The sheaves $\mathcal{F}$ and $\mathcal{F}_{\circ}$ are the main objects of study of the variational calculus. The sheaves $\mathbb{F}$ and $\mathbb{F}_{\circ}$ may be used to study $\mathcal{F}$ and $\mathcal{F}_{\circ}$, by the following algebraic Poincaré lemma. 
Theorem 2.1. Suppose that $U \subset \mathbb{R}^{n}$ and the body of $M$ are star-shaped. Then the complexes

$$
\begin{aligned}
0 & \rightarrow \mathbb{F}^{-n-1, *}\left(U \times T^{*}[-1] M\right) \stackrel{d}{\rightarrow} \mathbb{F}^{-n, *}\left(U \times T^{*}[-1] M\right) \stackrel{d}{\rightarrow} \cdots \\
& \stackrel{d}{\rightarrow} \mathbb{F}^{-1, *}\left(U \times T^{*}[-1] M\right) \stackrel{d}{\rightarrow} \mathbb{F}^{0, *}\left(U \times T^{*}[-1] M\right) \stackrel{\int}{\rightarrow} \mathcal{F}\left(U \times T^{*}[-1] M\right) \rightarrow 0,
\end{aligned}
$$

and

$$
\begin{aligned}
0 \rightarrow \mathbb{F}_{\circ}^{-n-1, *}\left(T^{*}[-1] M\right) & \stackrel{d}{\rightarrow} \mathbb{F}_{\circ}^{-n, *}\left(T^{*}[-1] M\right) \stackrel{d}{\rightarrow} \cdots \\
& \stackrel{d}{\rightarrow} \mathbb{F}_{\circ}^{-1, *}\left(T^{*}[-1] M\right) \stackrel{d}{\rightarrow} \mathbb{F}_{\circ}^{0, *}\left(T^{*}[-1] M\right) \stackrel{\int}{\rightarrow} \mathcal{F}_{\circ}\left(T^{*}[-1] M\right) \rightarrow 0,
\end{aligned}
$$

are exact.

We will need some of the details of the proof of this theorem. The proof in Olver [10, Theorem 5.56, (5.109), (5.111)] is based on explicit formulas (apparently due to Anderson) for a homotopy operator

$$
\mathrm{H}: \mathbb{F}_{\circ}^{-p, q} \rightarrow \mathbb{F}_{\circ}^{-p-1, q}
$$

and an operator

$$
\mathrm{P}: \mathcal{F}_{\circ} \rightarrow \mathbb{F}_{\circ}^{0, *}
$$

such that the operators $\int \mathrm{P}: \mathcal{F}_{\circ} \rightarrow \mathcal{F}_{\circ}$,

$$
\mathrm{H} d+\mathrm{P} \int: \mathbb{F}_{\circ}^{0, *} \rightarrow \mathbb{F}_{\circ}^{0, *}
$$

and

$$
d \mathrm{H}+\mathrm{H} d: \mathbb{F}_{\circ}^{-p, *} \rightarrow \mathbb{F}_{\circ}^{-p, *}, \quad p>0,
$$

all equal the identity. The operator $\mathbf{H}$ is given on $f \in \mathbb{F}_{\circ}^{-p, *}, 0 \leq p<n$, by the formula

$\mathrm{H} f=\sum_{i=1}^{n} \sigma_{i} \sum_{0<\beta \leq \alpha} \frac{\beta_{i}}{|\beta|+n-p}\left(\begin{array}{c}\alpha \\ \beta\end{array}\right) \partial^{\beta-i} \int_{0}^{1}\left(x^{a}(-\partial)^{\alpha-\beta} \partial_{a, \alpha} f(\lambda u)+\xi_{a}(-\partial)^{\alpha-\beta} \partial_{\alpha}^{a} f(\lambda u)\right) \frac{d \lambda}{\lambda}$, and on $\mathbb{F}_{\circ}^{-n-1,0}$ by $H \eta=\sigma_{1} \ldots \sigma_{n} \in \mathbb{F}^{-n, 0}$. The operator $\mathrm{P}$ is given on $\int f \in \mathcal{F}_{\circ}$, by the formula

$$
\mathrm{P} \int f=\int_{0}^{1}\left(x^{a} \delta_{a} f(\lambda u)+\xi_{a} \delta^{a} f(\lambda u)\right) \frac{d \lambda}{\lambda} .
$$

Filter $\mathbb{F}_{\circ}$ by the subspaces

$$
F^{p} \mathbb{F}_{\circ}=\sum_{r \geq p} \mathbb{F}_{\circ}^{-r, *}
$$

The operator $\mathrm{H}$ takes $F^{p} \mathbb{F}_{\circ}$ to $F^{p+1} \mathbb{F}_{\circ}$, and $F^{p} \mathbb{F}_{\circ}=0$ for $p>n+1$, and satisfies the formula $d \mathrm{H}+\mathrm{H} d=1$ on $F^{1} \mathbb{F}_{\text {o }}$.

The proof of Theorem 2.1 for $\mathbb{F}$ uses a more complicated homotopy $\mathrm{H}^{*}$, which is the sum of a homotopy $\mathrm{H}$ given by the same expression as for $\mathbb{F}_{0}$, plus the homotopy for differential forms on the star-shaped set $U$ that figures in the usual proof of the Poincaré lemma. The corresponding projection $\mathrm{P}: \mathcal{F} \rightarrow \mathbb{F}^{0, *}$ is given by the same expression as for $\mathcal{F}_{0} \rightarrow \mathbb{F}_{0}^{0, *}$. The homotopy $\mathrm{H}^{*}$ again satisfies the formula $d \mathrm{H}^{*}+\mathrm{H}^{*} d=1$ on $F^{1} \mathbb{F}$. 
The sheaf $\mathcal{F}$ is a graded Lie superalgebra with respect to the Batalin and Vilkovisky (anti)bracket

$$
\left(\int f, \int g\right)=\sum_{a}(-1)^{(\mathrm{p}(f)+1) \mathrm{p}\left(\xi_{a}\right)} \int\left(\delta_{a} f \delta^{a} g+(-1)^{\mathrm{p}(f)} \delta^{a} f \delta_{a} g\right) .
$$

A classical field theory is a solution $\int S \in \mathcal{F}$, of ghost number 0 and even parity, to the classical master equation (Maurer-Cartan equation) of Batalin and Vilkovisky:

$$
\frac{1}{2}\left(\int S, \int S\right)=0
$$

We refer to $\int S$ as the action of the theory. The operator

$$
\mathrm{s} \int f=\left(\int S, \int f\right)
$$

makes $\mathcal{F}$ into a differential graded Lie superalgebra; the cohomology $H^{*}(\mathcal{F}, \mathrm{s})$ is a graded Lie superalgebra, called the BV cohomology of the theory. (Some authors refer to this as the BRST cohomology.) The Batalin-Vilkovisky antibracket preserves the subsheaf $\mathcal{F}_{\circ} \subset \mathcal{F}$. We restrict attention in this paper to classical field theories whose action $\int S \in \mathcal{F}_{\circ}$ has no explicit dependence on the independent variables $\left\{t^{i}\right\}$.

The resolution $\mathbb{F}$ of $\mathcal{F}$ is a differential graded Lie superalgebra, with respect to a bracket introduced by Soloviev [11], and adapted to the Batalin-Vilkovisky formalism (at least, when $n=1$ ) in [6, 7]. This (anti)bracket is given by the explicit formula

$$
\begin{aligned}
\left(\left(\sigma_{I} f_{I}, \sigma_{J} g_{J}\right)\right)= & \sum_{a}(-1)^{\left(\mathrm{p}\left(f_{I}\right)+1\right)\left(\mathrm{p}\left(x_{a}\right)+|J|\right)} \\
& \sigma_{I} \sigma_{J} \sum_{\alpha, \beta \in \mathbb{N}^{n}}\left(\partial^{\beta} \partial_{a, \alpha} f_{I} \partial^{\alpha} \partial_{\beta}^{a} g_{J}+(-1)^{\mathrm{p}\left(f_{I}\right)} \partial^{\beta} \partial_{\alpha}^{a} f_{I} \partial^{\alpha} \partial_{a, \beta} g_{J}\right) .
\end{aligned}
$$

The Soloviev (anti)bracket preserves the subsheaf $\mathbb{F}_{\circ} \subset \mathbb{F}$.

Proposition 2.2. Given a classical field theory, represented by a solution $\int S \in \mathcal{F}_{\circ}$ of the classical master equation, there is a lift $S \in \mathbb{F}_{\circ}$ of $\int S$ to a solution of the Maurer-Cartan equation in $\mathbb{F}_{\circ}$ :

$$
d S+\frac{1}{2}((S, S))=0 .
$$

Proof. Since $\frac{1}{2}\left(\int S_{0}, \int S_{0}\right)=0 \in \mathcal{F}_{\circ}$, we see that there exists an element $S_{1} \in \mathbb{F}_{\circ}^{-1,1}$ such that

$$
d S_{1}+\frac{1}{2}\left(\left(S_{0}, S_{0}\right)\right)=0 .
$$

We now define $S_{k} \in \mathbb{F}_{\circ}^{-k, k}$ for $k>1$ by the recursive formula

$$
S_{k}=-\frac{1}{2} \sum_{j=0}^{k-1} \mathrm{H}\left(\left(S_{j}, S_{k-j-1}\right)\right) .
$$

Equivalently, $S$ is the fixed point of the equation

$$
S=S_{0}+S_{1}-\frac{1}{2} \mathrm{H}\left(((S, S))-\left(\left(S_{0}, S_{0}\right)\right)\right) .
$$


Let $T=d S+\frac{1}{2}((S, S))$. We see that

$$
d S=d S_{0}+d S_{1}-\frac{1}{2}\left(((S, S))-\left(\left(S_{0}, S_{0}\right)\right)\right)+\frac{1}{2} \mathrm{H}\left(d((S, S))-d\left(\left(S_{0}, S_{0}\right)\right)\right) .
$$

Since $d S_{0}=0$, this equals

$$
d S=d S_{1}+\left(\left(S_{0}, S_{0}\right)\right)-\frac{1}{2}((S, S)) \mathrm{H}((S, d S)) .
$$

By (2.3), we see that

$$
T=-\mathrm{H}((S, d S)) .
$$

By the Jacobi relation for the Soloviev bracket, $((S,((S, S))))=0$, hence

$$
T=-\mathrm{H}((S, T)) \text {. }
$$

Since $\mathrm{H}: F^{p} \mathbb{F}_{\circ} \rightarrow F^{p+1} \mathbb{F}_{\circ}$ raises filtration degree in $\mathbb{F}_{\circ}$, we see, by induction on $p$, that $T$ lies in $F^{p} \mathbb{F}_{\circ}$. Since $F^{p} \mathbb{F}_{\circ}$ vanishes if $p>n+1$, we conclude that $T=0$, proving the theorem.

We call $S$ the Lagrangian density of the theory. The lift of $S$ to $\mathbb{F}_{\circ}$ yields insight into the problem of extending field theories to manifolds with boundary, and possibly corners. For a different approach to these questions, see Cattaneo et al. [5].

Define an operator

$$
\mathrm{s} f=((S, f))
$$

on $\mathbb{F}$. By the classical master equation (2.2), the operator $d+\mathrm{s}$ on $\mathbb{F}$ is a differential,

$$
[d, \mathrm{~s}]+\mathrm{s}^{2}=0,
$$

and gives a lift of the differential $\mathbf{s}$ on $\mathcal{F}$ to $\mathbb{F}$. Equipped with this differential, $\mathbb{F}$ becomes a sheaf of differential graded Lie algebras.

Proposition 2.3. The morphism of sheaves of differential graded Lie superalgebras from $(\mathbb{F}, d+\mathrm{s})$ to $(\mathcal{F}, \mathrm{s})$ given by $f \mapsto \int f$ is a quasi-isomorphism.

The operator $\mathrm{s}$ is a graded derivation of the graded Lie algebra $\mathbb{F}$. There is another operator $\tilde{\mathrm{s}}$ defined using $S$ that is a graded derivation of the product on $\mathbb{F}$, namely the Hamiltonian vector field $\mathrm{X}_{S}$ associated to $S$. The (Batalin-Vilkovisky) Hamiltonian vector field associated to an element $f \in \mathbb{F}$ is the evolutionary vector field given by the formula

$$
\mathrm{X}_{f}(g)=\sum_{I, J \in \mathbb{N}^{n}} \mathrm{X}_{\sigma_{I} f_{I}}\left(\sigma_{J} g_{J}\right),
$$

where

$$
\begin{aligned}
\mathrm{X}_{\sigma_{I} f_{I}}\left(\sigma_{J} g_{J}\right)= & \sum_{a}(-1)^{\left(\mathrm{p}\left(f_{I}\right)+1\right)\left(\mathrm{p}\left(x_{a}\right)+|J|\right)} \sigma_{I} \sigma_{J} \operatorname{pr}\left(\delta_{a, \alpha} f_{I} \partial^{a} g_{J}+(-1)^{\mathrm{p}\left(f_{I}\right)} \delta_{\alpha}^{a} f_{I} \partial_{a} g_{J}\right) \\
= & \sum_{a}(-1)^{\left(\mathrm{p}\left(f_{I}\right)+1\right)\left(\mathrm{p}\left(x_{a}\right)+|J|\right)} \\
& \sigma_{I} \sigma_{J} \sum_{\alpha, \beta \in \mathbb{N}^{n}}(-1)^{|\alpha|}\left(\partial^{\alpha+\beta} \partial_{a, \alpha} f_{I} \partial_{\beta}^{a} g_{J}+(-1)^{\mathrm{p}\left(f_{I}\right)} \partial^{\alpha+\beta} \partial_{\alpha}^{a} f_{I} \partial_{a, \beta} g_{J}\right) .
\end{aligned}
$$


To $f \in \mathbb{F}$, we have now associated two different operators on $\mathbb{F}$, namely the infinite-order differential operator $\operatorname{ad}(f)$ and the vector field $\mathbf{X}_{f}$. It turns out that these operators are homotopic.

Lemma 2.4. Let

$$
\begin{aligned}
& C\left(\sigma_{I} f_{I}, \sigma_{J} g_{J}\right)=\sum_{i=1}^{n} \sum_{a}(-1)^{\left(\mathrm{p}\left(f_{I}\right)+1\right)\left(\mathrm{p}\left(x_{a}\right)+|J|\right)} \sigma_{i} \sigma_{I} \sigma_{J} \\
& \quad \sum_{\alpha, \beta \in \mathbb{N}^{n}} \sum_{0<\gamma \leq \alpha} \frac{\gamma_{i}}{|\gamma|}\left(\begin{array}{l}
\alpha \\
\gamma
\end{array}\right)(-\partial)^{\gamma-i}\left(\partial^{\beta} \partial_{a, \alpha} f_{I} \partial^{\alpha-\gamma} \partial_{\beta}^{a} g_{J}+(-1)^{\mathrm{p}\left(f_{I}\right)} \partial^{\beta} \partial_{\alpha}^{a} f_{I} \partial^{\alpha-\gamma} \partial_{a, \beta} g_{J}\right),
\end{aligned}
$$

where $\gamma-i$ is the multi-index $\left(\gamma_{1}, \ldots, \gamma_{i}-1, \ldots, \gamma_{n}\right)$. Then

$$
d C(f, g)=\mathrm{X}_{f} g-((f, g))+C(d f, g)+(-1)^{\mathrm{p}(f)} C(f, d g)
$$

Proof. This is a consequence of the formula

$$
\begin{aligned}
& \left(\mathrm{X}_{\sigma_{I} f_{I}}-\operatorname{ad}\left(\sigma_{I} f_{I}\right)\right)\left(\sigma_{J} g_{J}\right)=\sum_{I} \sum_{a}(-1)^{\left(\mathrm{p}\left(f_{I}\right)+1\right)\left(\mathrm{p}\left(x_{a}\right)+|J|\right)} \\
& \sigma_{I} \sigma_{J} \sum_{\alpha, \beta \in \mathbb{N}^{n}} \sum_{0<\gamma \leq \alpha}\left(\begin{array}{c}
\alpha \\
\gamma
\end{array}\right)(-\partial)^{\gamma}\left(\partial^{\beta} \partial_{a, \alpha} S_{I} \partial^{\alpha-\gamma} \partial_{\beta}^{a} g_{J}+(-1)^{\mathrm{p}\left(f_{I}\right)} \partial^{\beta} \partial_{\alpha}^{a} S_{I} \partial^{\alpha-\gamma} \partial_{a, \beta} g_{J}\right) .
\end{aligned}
$$

Consider the operator

$$
\tilde{\mathrm{s}}=\mathrm{X}_{S}=\sum_{I} \mathrm{X}_{\sigma_{I} S_{I}}
$$

The following theorem, relating the action of the operators $\mathrm{s}$ and $\tilde{s}$, is the first main result of this paper.

Theorem 2.5. Suppose that $U \subset \mathbb{R}^{n}$ and the body of $M$ is star-shaped. Then there is an automorphism

$$
\alpha=1+\sum_{k=1}^{\infty} \alpha_{k}
$$

where $\alpha_{k}: \mathbb{F}^{p, q}\left(U \times T^{*}[-1] M\right) \rightarrow \mathbb{F}^{p-k, q+k}\left(U \times T^{*}[-1] M\right)$ is an operator of even parity, that intertwines the operators $d+\mathrm{s}$ and $d+\tilde{\mathrm{s}}$ :

$$
(d+\tilde{\mathbf{s}}) \alpha=\alpha(d+\mathrm{s})
$$

Note that $\alpha$ is actually a finite sum, since the operators $\alpha_{k}$ vanish for $k>n+1$.

The following corollary also follows from results contained in [10].

Corollary 2.6. The operator $d+\tilde{\mathbf{s}}$ is a differential of the sheaf of graded superspaces $\mathbb{F}$, and there is a natural isomorphism $H^{*}(\mathbb{F}, d+\mathrm{s}) \cong H^{*}(\mathbb{F}, d+\tilde{\mathbf{s}})$.

Since $\tilde{\mathbf{s}}$ is evolutionary, we have $[d, \tilde{\mathbf{s}}]=0$, and hence the above corollary actually establishes that $\tilde{\mathbf{s}}^{2}=0$. Another proof of this result is given in [7] for $n=1$. 
Proof of Theorem 2.5. Write

$$
S_{n}=\sum_{|I|=k} \sigma_{I} S_{I}, \quad \tilde{\mathrm{s}}_{k}=\sum_{|I|=k} \mathrm{X}_{\sigma_{I} S_{I}}, \quad \mathrm{~s}_{n}=\sum_{|I|=k} \operatorname{ad}\left(\sigma_{I} S_{I}\right) .
$$

The operator $\alpha_{1}$ must satisfy the equation

$$
\left[d, \alpha_{1}\right]+\tilde{\mathbf{s}}_{0}-\mathrm{s}_{0}=0 .
$$

By Lemma 2.4, we may choose $\alpha_{1} f=C\left(S_{0}, f\right)$.

The operators $\alpha_{n}, n>1$, satisfy the recursion

$$
\left[d, \alpha_{k}\right]+\sum_{i=0}^{k-2}\left(\tilde{\mathbf{s}}_{i} \alpha_{k-i-1}-\alpha_{k-i-1} \mathbf{s}_{i}\right)+\tilde{\mathbf{s}}_{k-1}-\mathrm{s}_{k-1}=0 .
$$

We make the Ansatz

$$
\alpha_{k}=\mathbf{H}^{*}\left(\sum_{i=0}^{k-1}\left(\tilde{\mathbf{s}}_{k} \alpha_{k-i-1}-\alpha_{k-i-1} \mathbf{s}_{i}\right)+\tilde{\mathbf{s}}_{k-1}-\mathbf{s}_{k-1}\right)
$$

The important point is that in this formula, $\mathrm{H}^{*}$ is only applied to elements of $F^{1} \mathbb{F}$, where the algebraic Poincaré lemma yields a contracting homotopy of the differential $d$.

To establish the theorem, we reformulate the recursion (2.4) as the solution of a fixed-point equation. We have

$$
\alpha=1+\alpha_{1}-\mathrm{H}^{*}\left(\tilde{\mathbf{s}} A-A \mathrm{~s}-\tilde{\mathbf{s}}_{0}+\mathbf{s}_{0}\right) .
$$

It is easily seen that this reformulates the recursive definition of the operators $\alpha_{k}$.

Let $\beta=[d, \alpha]+\tilde{\mathbf{s}} \alpha-\alpha$ s. Since $\mathbf{H}^{*}$ is being applied to elements of $F^{1} \mathbb{F}$ in this expression, we may set $\left[d, \mathrm{H}^{*}\right]$ equal to 1 in calculating $[d, \alpha]$. A straightforward calculation now shows that

$$
\begin{aligned}
{[d, \alpha]=} & {\left[d, \alpha_{1}\right]-\left[d, \mathbf{H}^{*}\right]\left(\tilde{\mathbf{s}} \alpha-\alpha \mathbf{s}-\tilde{\mathbf{s}}_{0}+\mathbf{s}_{0}\right) } \\
& +\mathbf{H}^{*}([d, \tilde{\mathbf{s}}] \alpha-\tilde{\mathbf{s}}[d, \alpha]-[d, \alpha] \mathbf{s}-\alpha[d, \mathbf{s}]) \\
= & \mathbf{s}_{0}-\tilde{\mathbf{s}}_{0}-\left(\tilde{\mathbf{s}} \alpha-\alpha \mathbf{s}-\tilde{\mathbf{s}}_{0}+\mathbf{s}_{0}\right)+\mathbf{H}^{*}\left(-\tilde{\mathbf{s}}^{2} \alpha-\tilde{\mathbf{s}}[d, \alpha]-[d, \alpha] \mathbf{s}+\alpha \mathbf{s}^{2}\right) \\
= & -(\tilde{\mathbf{s}} \alpha-\alpha \mathbf{s})+\mathbf{H}^{*}\left(-\tilde{\mathbf{s}}^{2} \alpha-\tilde{\mathbf{s}}[d, \alpha]-[d, \alpha] \mathbf{s}+\alpha \mathbf{s}^{2}\right),
\end{aligned}
$$

in other words,

$$
\beta=-\mathbf{H}^{*}(\tilde{\mathbf{s}} \beta-\beta \mathbf{s}) .
$$

We see, by induction on $p$, that $\beta$ maps $\mathbb{F}$ to $F^{p} \mathbb{F}$. Since $F^{p} \mathbb{F}$ vanishes if $p>n+1$, we conclude that $\beta=0$, proving the theorem.

The resolution $(\mathbb{F}, d+\tilde{\mathbf{s}})$ of the complex $(\mathcal{F}, \mathrm{s})$ has the disadvantage of not being a differential graded Lie superalgebra in any natural way. It does present some technical advantages over the sheaf of differential graded Lie superalgebras $(\mathbb{F}, d+\tilde{\mathbf{s}})$, since it is the total complex of a bicomplex with differentials $d$ and $\tilde{\mathbf{s}}$, and there are many methods available to study the cohomology of the evolutionary vector field $\tilde{s}$. 


\section{Covariant field theories}

Introduce formal variables $\left\{u^{1}, \ldots, u^{n}\right\}$ of ghost number $\operatorname{gh}\left(u^{i}\right)=2$, corresponding to the coefficients of a vector field $u^{i} \partial_{i}$ on $\mathbb{R}^{n}$. Define the densities

$$
D_{i}=\left\langle\partial_{i} x^{a}, \xi_{a}\right\rangle, \quad i \in\{1, \ldots, n\},
$$

and let $D_{u}=u^{i} D_{i}$.

A covariant classical field theory is a solution $\int S_{u} \in \mathcal{F}_{\circ} \llbracket u \rrbracket$, of total ghost number 0 and even parity, to the covariant extension of the classical master equation

$$
\frac{1}{2}\left(\left(\int S_{u}, \int S_{u}\right)\right)=\int D_{u} .
$$

In particular, setting the variables $u^{i}$ to zero, we recover a solution of the classical master equation.

Lemma 3.1. The operator

$$
\iota_{i}=\sigma_{i}\left(1-\sum_{\alpha \in \mathbb{N}^{n}} \xi_{a, \alpha} \partial_{a, \alpha}\right)
$$

satisfies the equations $\iota_{i} D_{j}=0$ and

$$
d \iota_{i}+\iota_{i} d+\operatorname{ad}\left(D_{i}\right)=0
$$

Proof. The equation $\iota_{i} D_{j}=0$ is clear by inspection, while the formula for $d \iota_{i}+\iota_{i} d$ is a special case of Lemma 2.4, since

$$
\mathrm{X}_{D_{i}}=-\partial_{i}
$$

and

$$
\sum_{\alpha \in \mathbb{N}^{n}} \xi_{a, \alpha} \partial_{a, \alpha}=C\left(D_{i},-\right)
$$

Define the covariant extension of the differential $d$ by the formula $d_{u}=d+\iota_{u}$, where $\iota_{u}=u^{i} \iota_{i}$. The analogue of Proposition 2.2 holds in this setting.

Proposition 3.2. Given a covariant classical field theory, represented by a solution $\int S_{u} \in$ $\mathcal{F}_{\circ} \llbracket u \rrbracket$ of the covariant classical master equation, there is a lift $S_{u} \in \mathbb{F}_{\circ} \llbracket u \rrbracket$ of $\int S_{u}$ to a solution of the curved Maurer-Cartan equation in $\mathbb{F}_{\circ} \llbracket u \rrbracket$ :

$$
d_{u} S_{u}+\frac{1}{2}\left(\left(S_{u}, S_{u}\right)\right)=D_{u}
$$

Proof. We expand $S_{u}$ as the sum

$$
S_{u}=\sum_{k=0}^{n+1} S_{k},
$$

where $S_{k} \in \mathbb{F}_{\circ}^{-k, *} \llbracket u \rrbracket$ has total degree 0 . Since $\frac{1}{2}\left(\int S_{0}, \int S_{0}\right)-\int D_{u}=0 \in \mathcal{F}_{\circ} \llbracket u \rrbracket$, we may find an element $S_{1} \in \mathbb{F}_{\circ}^{-1, *} \llbracket u \rrbracket$ of total degree 0 such that

$$
d S_{1}+\frac{1}{2}\left(\left(S_{0}, S_{0}\right)\right)=D_{u}
$$


We now define $S_{k} \in \mathbb{F}_{\circ}^{-k, *} \llbracket u \rrbracket$ for $k>1$ by the recursive formula

$$
S_{k}=-\mathrm{H}\left(\iota_{u} S_{k-1}+\frac{1}{2} \sum_{j=0}^{k-1}\left(\left(S_{j}, S_{k-j-1}\right)\right)\right) .
$$

It is vital to the proof that the argument of $\mathrm{H}$ lies in $F^{1} \mathbb{F}_{\circ}$, where $[d, \mathrm{H}]=1$.

Equivalently, $S$ is the fixed point of the equation

$$
S_{u}=S_{0}+S_{1}-\mathrm{H}\left(\iota_{u} S_{u}+\frac{1}{2}\left(\left(S_{u}, S_{u}\right)\right)-\frac{1}{2}\left(\left(S_{0}, S_{0}\right)\right)\right) .
$$

Since $d S_{0}=0$, we see that

$$
\begin{aligned}
d S_{u} & =d S_{1}-\left(\iota_{u} S_{u}+\frac{1}{2}\left(\left(S_{u}, S_{u}\right)\right)-\frac{1}{2}\left(\left(S_{0}, S_{0}\right)\right)\right)+\mathrm{H} d\left(\iota_{u} S_{u}+\frac{1}{2}\left(\left(S_{u}, S_{u}\right)\right)-\frac{1}{2}\left(\left(S_{0}, S_{0}\right)\right)\right) \\
& =d S_{1}+\frac{1}{2}\left(\left(S_{0}, S_{0}\right)\right)-\iota_{u} S_{u}-\frac{1}{2}\left(\left(S_{u}, S_{u}\right)\right)+\mathrm{H}\left(d \iota_{u} S_{u}-\left(\left(S_{u}, d S_{u}\right)\right)\right) .
\end{aligned}
$$

Let $T_{u}=d_{u} S_{u}+\frac{1}{2}\left(\left(S_{u}, S_{u}\right)\right)-D_{u}$. We find that

$$
T_{u}=-\mathrm{H}\left(\iota_{u} T_{u}+\left(\left(S_{u}, T_{u}\right)\right)\right)
$$

Here, we have used the Jacobi relation for the Soloviev bracket, in the form $\left(\left(S_{u},\left(\left(S_{u}, S_{u}\right)\right)\right)\right)=$ 0 . Since $\mathrm{H}: F^{p} \mathbb{F}_{\circ} \rightarrow F^{p+1} \mathbb{F}_{\circ}$ raises filtration degree in $\mathbb{F}_{\circ}$, we see, by induction on $p$, that $T_{u}$ lies in $F^{p} \mathbb{F}_{\circ} \llbracket u \rrbracket$. Since $F^{p} \mathbb{F}_{\circ}$ vanishes if $p>n+1$, we conclude that $T_{u}=0$, proving the theorem.

Remark 3.3. Just as the usual Maurer-Cartan equation is an abstracted form of the equation for a flat connection, the curved Maurer-Cartan equation may be thought of as an abstraction for the equation for a projectively flat connection.

Introduce the operator $\mathrm{s}_{u}=\operatorname{ad}\left(S_{u}\right)$. It follows from Lemma 3.1 that if $S_{u}$ satisfies the covariant classical master equation, then $d_{u}+\mathrm{s}_{u}$ is a differential on $\mathbb{F} \llbracket u \rrbracket$. We now restrict attention to the special case where $S_{u}$ is an affine function of the variables $u^{i}$ :

$$
S_{u}=S+u^{i} G_{i}
$$

Note that $G_{i} \in \mathbb{F}_{\circ}$ has ghost number -2 and even parity. The equation (3.1) is equivalent to the classical master equation (2.2) for $S$ and the two system of equations

$$
d G_{i}+\iota_{i} S+\left(\left(S, G_{i}\right)\right)=D_{i} \quad 1 \leq i \leq n,
$$

and

$$
\iota_{i} G_{j}+\iota_{j} G_{i}+\left(\left(G_{i}, G_{j}\right)\right)=0, \quad 1 \leq i, j \leq n .
$$

Introduce the (degree -1 , odd parity) operators

$$
\Gamma_{i}=\iota_{i}+\operatorname{ad}\left(G_{i}\right)
$$


on $\mathbb{F}$. These pairwise graded commute (i.e. anticommute) with each other and with the differential $d+\mathrm{s}$, and we have

$$
d_{u}+\mathrm{s}_{u}=d+\mathrm{s}+u^{i} \Gamma_{i}
$$

The following theorem gives a purely algebraic transcription of the construction of the Lagrangian density of AKSZ models in the framework of covariant field theories. Let $\Gamma=\Gamma_{1} \ldots \Gamma_{n}$.

Theorem 3.4. Let $\rho \in \mathbb{F}_{\circ}^{0, n}$ be a cocycle for the differential $\mathrm{s}$, such that $((\rho, \Gamma \rho))=0$. Then

$$
S_{u}^{\rho}=S_{u}+\Gamma \rho
$$

is a solution of the covariant classical master equation (3.1).

Proof. By the equations $\mathrm{s} \rho=(d+\mathrm{s}) \rho=0$ and $\left[d+\mathrm{s}, \Gamma_{i}\right]=0$, it follows that

$$
\left(d_{u}+\mathbf{s}_{u}\right) \Gamma \rho=u^{i} \Gamma_{i}(\Gamma \rho) .
$$

By the equation $\Gamma^{i} \Gamma^{j}+\Gamma^{j} \Gamma^{i}=0$, we see that $\Gamma_{i}(\Gamma \rho)=\Gamma_{i}\left(\Gamma_{1} \ldots n \rho\right)=0$, and hence that

$$
\left(d_{u}+\mathbf{s}_{u}\right) \Gamma \rho=0 .
$$

Since $\Gamma_{i}$ is a graded derivation of the Soloviev bracket and $\Gamma_{i}(\Gamma \rho)=0$, we see that

$$
\Gamma((\rho, \Gamma \rho))=((\Gamma \rho, \Gamma \rho)),
$$

showing that

$$
((\Gamma \rho, \Gamma \rho))=0,
$$

and completing the proof.

Since the induced action of $\Gamma_{i}$ on $\mathcal{F}$ equals ad $\left(\int G_{i}\right)$, we obtain the following corollary.

Corollary 3.5. Let $\int \rho \in \mathcal{F}_{\circ}^{n}$ be a cocycle for the differential s, of ghost number and parity $n$, such that $\left(\left(\int G_{1}, \ldots,\left(\int G_{n}, \int \rho\right) \ldots\right), \int \rho\right)=0$. Then

$$
\int S^{\rho}=\int S+\left(\int G_{1}, \ldots,\left(\int G_{n}, \int \rho\right) \ldots\right) \in \mathcal{F}_{\circ}
$$

is a solution of the classical master equation (2.1).

The remainder of this section is not used elsewhere in the article, but serves to relate the above formulas to the approach of [7], which handled the special case where $n=1$. In loc. cit., we expressed the twist of a solution of the covariant classical master equation by a cochain $\rho$ as a gauge transformation.

If $W \in \mathbb{F}_{\circ}[u]$ has total ghost number -1 , it generates a gauge transformation of solutions of the covariant classical master equation:

$$
S_{u} \bullet W=S_{u}+\sum_{k=0}^{\infty} \frac{1}{(k+1) !}(-\operatorname{ad}(W))^{k}\left(d_{u}+\mathrm{s}_{u}\right) W .
$$

If $W$ satisfies the auxilliary equation $\left(\left(W,\left(d_{u}+\mathrm{s}_{u}\right) W\right)\right)=0$, then $S_{u} \bullet \rho=S_{u}+\left(d_{u}+\mathrm{s}_{u}\right) W$. 
More generally, this formula still applies if $W \in \mathbb{F}_{\circ}(u)$ is a rational function of the variables $u^{i}$ such that $\left(d_{u}+\mathbf{s}_{u}\right) W \in \mathbb{F}_{\circ}[u]$ is a polynomial function of the variables $u^{i}$ and $\left(\left(W,\left(d_{u}+\mathbf{s}_{u}\right) W\right)\right)=0$. This way of constructing solutions of the Maurer-Cartan equation is a nonlinear analogue of the idea that a coboundary in a complex is a cocycle in any subcomplex that contains it. We may express the solution $S_{u}+\Gamma \rho$ of the covariant classical master equation as a singular gauge transformation of $S_{u}$.

Make the Ansätz

$$
W=\frac{1}{n !(u \cdot u)} \epsilon_{i_{1}}^{i_{2} \ldots i_{n}} u^{i_{1}} \Gamma_{i_{2}} \ldots \Gamma_{i_{n}} \rho,
$$

where $u \cdot u=\delta_{i j} u^{i} u^{j}$. It follows that $\left(d_{u}+\mathbf{s}_{u}\right) W=\Gamma \rho$, and that

$$
\begin{aligned}
\left(\left(W,\left(d_{u}+\mathrm{s}_{u}\right) W\right)\right) & =\frac{1}{n !(u \cdot u)} \epsilon_{i_{1}}{ }^{i_{2} \ldots i_{n}} u^{i_{1}}\left(\left(\Gamma_{i_{2}} \ldots \Gamma_{i_{n}} \rho, \Gamma \rho\right)\right) \\
& =\frac{1}{n !(u \cdot u)} \epsilon_{i_{1}}{ }^{i_{2} \ldots i_{n}} u^{i_{1}} \Gamma_{i_{2}} \ldots \Gamma_{i_{n}}((\rho, \Gamma \rho))=0 .
\end{aligned}
$$

We see that $S_{u}^{\rho}=S_{u} \bullet W$ is obtained from $S_{u}$ by a singular gauge transformation of Maurer-Cartan elements in the curved Lie algebra $\left(\mathbb{F}_{\circ}[u], d_{u}+\mathbf{s}_{u}\right)$.

\section{The covariant abelian Chern-Simons action}

In the remainder of this paper, we consider the special case of classical Chern-Simons theory. This covariant field theory is quite special: it is a first-order field theory in which every physical field is bosonic. In this section, we solve the covariant classical master equation for abelian Chern-Simons theory, and calculate its Batalin-Vilkovisky cohomology. We build upon these results in the next section, where we solve the covariant classical master equation for non-abelian classical Chern-Simons theory.

The lift of the action for the abelian Chern-Simons field theory to $\mathbb{F}_{\circ}$ is

$$
\Pi=\frac{1}{2} \epsilon^{i j k}\left\langle A_{i}, \partial_{j} A_{k}\right\rangle+\frac{1}{2}\left\langle\partial_{i} c, A^{+i}\right\rangle-\frac{1}{2}\left\langle c, \partial_{i} A^{+i}\right\rangle \in \mathbb{F}_{\circ}^{0,0}
$$

It is clear that $d \Pi=0$, and that $\iota_{i} \Pi=0$ for $1 \leq i \leq n$. The classical master equation follows on verification that $\frac{1}{2}((\Pi, \Pi))=0$, which is established by a straightforward calculation. Let $\pi=\operatorname{ad}(\Pi)$, and $\tilde{\pi}=\mathrm{X}_{\Pi}$.

Define evolutionary vector fields

$$
\mathrm{f}^{i}=\frac{1}{2}\left(-\left\langle c, \frac{\partial}{\partial A_{i}}\right\rangle+\epsilon^{i j k}\left\langle A_{j}, \frac{\partial}{\partial A^{+k}}\right\rangle+\left\langle A^{+i}, \frac{\partial}{\partial c^{+}}\right\rangle\right), \quad 1 \leq i \leq 3 .
$$

(These vector fields are not Hamiltonian.) It is easily seen that $\left[\pi, f^{i}\right]=\left[f^{i}, f^{j}\right]=0$ for $1 \leq i, j \leq 3$. The following result shows that for the abelian Chern-Simons theory, there is a particularly simple choice for the automorphism $\alpha$ of $\mathbb{F}$ introduced in Theorem 2.5. 


\begin{tabular}{|c|ll|}
\hline 0 & $\partial^{\alpha} c^{+}$ & \\
& $\partial^{\alpha} A^{+1}$ & $\alpha_{1}>0$ \\
\hline 1 & $\partial^{\alpha} A^{+1}$ & $\alpha_{1}=0$ \\
& $\partial^{\alpha} A^{+2}$ & \\
& $\partial^{\alpha} A^{+3}$ & \\
& $\partial^{\alpha} A_{2}$ & $\alpha_{1}>0$ \\
& $\partial^{\alpha} A_{3}$ & $\alpha_{1}+\alpha_{2}>0$ \\
\hline 2 & $\partial^{\alpha} A_{1}$ & \\
& $\partial^{\alpha} A_{2}$ & $\alpha_{1}=0$ \\
& $\partial^{\alpha} A_{3}$ & $\alpha_{1}+\alpha_{2}=0$ \\
& $\partial^{\alpha} c$ & $\alpha_{1}+\alpha_{2}+\alpha_{3}>0$ \\
\hline 3 & $c$ & \\
\hline
\end{tabular}

Table 1. Filtration of jet coordinates.

Proposition 4.1. $\exp \left(\sigma_{i} \mathrm{f}^{i}\right)(d+\tilde{\pi})=(d+\pi) \exp \left(\sigma_{i} \mathrm{f}^{i}\right)$

Proof. Observe that $C(\Pi,-)=-\sigma_{i} \mathrm{f}^{i}$. It follows that

$$
\begin{aligned}
\pi & =\tilde{\pi}-[d, C(\Pi,-)] \\
& =\tilde{\pi}+\partial_{i} \mathrm{f}^{i} .
\end{aligned}
$$

The proof is completed by observing that

$$
\left[\left[d, \sigma_{i} \mathrm{f}^{i}\right], \sigma_{j} \mathrm{f}^{j}\right]=\left[\pi, \sigma_{i} \mathrm{f}^{i}\right]=0 .
$$

The Batalin-Vilkovisky cohomology $H^{*}(\mathcal{F}, \pi)$ of the abelian Chern-Simons theory is easily calculated. We must calculate the cohomology of the complex $\left(\mathbb{F}^{*}, d+\pi\right)$, which by Proposition 4.1 is isomorphic to the cohomology of the double complex $\left(\mathbb{F}^{*, *}, d, \tilde{\pi}\right)$. We take the cohomology of $\tilde{\pi}$ first. The vector field $\tilde{\pi}$ is given by the formula

$$
\tilde{\pi}=\operatorname{pr}\left(-\left\langle\partial_{i} c, \frac{\partial}{\partial A_{i}}\right\rangle+\epsilon^{i j k}\left\langle\partial_{i} A_{j}, \frac{\partial}{\partial A^{+k}}\right\rangle+\left\langle\partial_{i} A^{+i}, \frac{\partial}{\partial c^{+}}\right\rangle\right) .
$$

Following the proof of Barnich et al. [2], we filter the jet coordinates according to Table 1. The leading order term of the differential $\tilde{\pi}$ in this filtration is

$$
\begin{gathered}
\sum_{\alpha}\left(\left\langle\partial^{\alpha+1} A^{+1}, \frac{\partial}{\partial\left(\partial^{\alpha} c^{+}\right)}\right\rangle+\left\langle\partial^{\alpha+1} A_{2}, \frac{\partial}{\partial\left(\partial^{\alpha} A^{+3}\right)}\right\rangle-\left\langle\partial^{\alpha+1} A_{3}, \frac{\partial}{\partial\left(\partial^{\alpha} A^{+2}\right)}\right\rangle-\left\langle\partial^{\alpha+1} c, \frac{\partial}{\partial\left(\partial^{\alpha} A_{1}\right)}\right\rangle\right) \\
+\sum_{\left\{\alpha \mid \alpha_{1}=0\right\}}\left(\left\langle\partial^{\alpha+2} A_{3}, \frac{\partial}{\partial\left(\partial^{\alpha} A^{+1}\right)}\right\rangle-\left\langle\partial^{\alpha+2} c, \frac{\partial}{\partial\left(\partial^{\alpha} A_{2}\right)}\right\rangle\right)-\sum_{\left\{\alpha \mid \alpha_{1}+\alpha_{2}=0\right\}}\left\langle\partial^{\alpha+3} c, \frac{\partial}{\partial\left(\partial^{\alpha} A_{3}\right)}\right\rangle,
\end{gathered}
$$

Taking cohomology, we obtain the graded vector space $C^{*}(\mathfrak{g})$ of cochains on the Lie algebra $\mathfrak{g}$, generated by $c$. The differential on $C^{*}(\mathfrak{g})$ vanishes, since $\mathfrak{g}$ is abelian. Denote by $\tau_{\geq 1} C^{*}(\mathfrak{g})$ the associated reduced complex, obtained by quotienting $C^{*}(\mathfrak{g})$ by $C^{0}(\mathfrak{g})$. 
The complex $\left(H^{*}(\mathbb{F}, \tilde{\pi}), d\right)$ may be identified with the augmented de Rham complex

$$
0 \rightarrow \mathbb{R} \eta \rightarrow \Omega^{0} \otimes C^{*}(\mathfrak{g}) \stackrel{d}{\rightarrow} \Omega^{1} \otimes C^{*}(\mathfrak{g}) \stackrel{d}{\rightarrow} \rightarrow \Omega^{2} \otimes C^{*}(\mathfrak{g}) \stackrel{d}{\rightarrow} \Omega^{3} \otimes C^{*}(\mathfrak{g}) \longrightarrow 0
$$

Applying the Poincaré lemma, we see that the $E_{2}$-page $H^{p}\left(H^{q}(\mathbb{F}, \tilde{\pi}), d\right)$ for the spectral sequence of the double complex equals

$$
H^{p}\left(H^{q}(\mathbb{F}, \tilde{\pi}), d\right) \cong \begin{cases}C^{q}(\mathfrak{g}), & p=-3, q>0 \\ 0, & \text { otherwise }\end{cases}
$$

Clearly, the spectral sequence is convergent (since $E_{0}^{p q}$ vanishes for $p$ outside the bounded interval $[-4,0]$ ), and collapses at the $E_{2}$-page. This proves the following theorem.

Theorem 4.2. If $\mathfrak{g}$ is abelian, the Batalin-Vilkovisky cohomology of the classical ChernSimons theory associated to $\mathfrak{g}$ equals

$$
H^{k}(\mathcal{F}, \pi) \cong \tau_{\geq 1} C^{k+3}(\mathfrak{g})
$$

We now prove that the abelian Chern-Simons theory is a covariant field theory; this will allow us to obtain explicit formulas for cocycles in $\mathcal{F}$ representing elements of the Batalin-Vilkovisky cohomology. The Chern-Simons theory treats the fields and antifields of the theory equally, as components of different degrees of a superconnection. It turns out that the formulas for the contravariant field theory are simplified if we use the following modification of $D_{i}$ which treats the fields and antifields equally:

$$
D_{i}=\frac{1}{2}\left\langle\partial_{i} A^{j}, A_{j}^{+}\right\rangle-\frac{1}{2}\left\langle A^{j}, \partial_{i} A_{j}^{+}\right\rangle+\frac{1}{2}\left\langle\partial_{i} c, c^{+}\right\rangle-\frac{1}{2}\left\langle c, \partial_{i} c^{+}\right\rangle, \quad i \in\{1, \ldots, n\} .
$$

The associated operator $\iota_{i}$ is given by the formula

$$
\begin{aligned}
\iota_{i} & =\sigma_{i}\left(1-C\left(D_{i},-\right)\right) \\
& =-\frac{1}{2} \sigma_{i}(\mathrm{E}-2),
\end{aligned}
$$

where $E$ is the Euler vector field, which in this setting equals

$$
\mathrm{E}=\operatorname{pr}\left(\left\langle A_{i}, \frac{\partial}{\partial A_{i}}\right\rangle+\left\langle A^{+i}, \frac{\partial}{\partial A^{+i}}\right\rangle+\left\langle c, \frac{\partial}{\partial c}\right\rangle+\left\langle c^{+}, \frac{\partial}{\partial c^{+}}\right\rangle\right)
$$

Let

$$
G_{i}=\frac{1}{2} \epsilon_{i j k}\left\langle A^{+j}, A^{+k}\right\rangle+\left\langle A_{i}, c^{+}\right\rangle \in \mathbb{F}_{\circ}^{0,-2} .
$$

It is clear that $d G_{i}=0$ for $1 \leq i \leq n$, and that $\iota_{i} G_{j}=0$ for $1 \leq i, j \leq n$.

Lemma 4.3. $\left(\left(\Pi, G_{i}\right)\right)=D_{i}$ 
Proof. In the proof, we make use of the formula $\delta_{r}^{k} \epsilon^{p q r} \epsilon_{i j k}=\delta_{i}^{p} \delta_{j}^{q}-\delta_{j}^{p} \delta_{i}^{q}$. We have

$$
\begin{aligned}
\left(\left(\epsilon^{p q r}\left\langle A_{p}, \partial_{q} A_{r}\right\rangle, G_{i}\right)\right) & =\left(\left(\epsilon^{p q r}\left\langle A_{p}, \partial_{q} A_{r}\right\rangle, \frac{1}{2} \epsilon_{i j k}\left\langle A^{+j} A^{+k}\right\rangle\right)\right) \\
& =\left(\delta_{i}^{q} \delta_{k}^{r}-\delta_{k}^{q} \delta_{i}^{r}\right)\left\langle\partial_{q} A_{r}, A^{+k}\right\rangle+\left(\delta_{i}^{p} \delta_{k}^{q}-\delta_{k}^{p} \delta_{i}^{q}\right)\left\langle A_{p}, \partial_{q} A^{+k}\right\rangle \\
& =\left\langle\partial_{i} A_{j}, A^{+j}\right\rangle-\left\langle\partial_{j} A_{i}, A^{+j}\right\rangle+\left\langle A_{i}, \partial_{j} A^{+j}\right\rangle-\left\langle A_{j}, \partial_{i} A^{+j}\right\rangle \\
\left(\left(\left\langle\partial_{p} c, A^{+p}\right\rangle, G_{i}\right)\right) & =\left(\left(\left\langle\partial_{p} c, A^{+p}\right\rangle,\left\langle A_{i}, c^{+}\right\rangle\right)\right) \\
& =\left\langle\partial_{i} c, c^{+}\right\rangle+\left\langle\partial_{j} A_{i}, A^{+j}\right\rangle, \\
\left(\left(\left\langle c, \partial_{p} A^{+p}\right\rangle, G_{i}\right)\right) & =\left(\left(\left\langle c, \partial_{p} A^{+p}\right\rangle,\left\langle A_{i}, c^{+}\right\rangle\right)\right) \\
& =\left\langle c, \partial_{i} c^{+}\right\rangle+\left\langle A_{i}, \partial_{j} A^{+j}\right\rangle .
\end{aligned}
$$

The lemma follows.

Corollary 4.4. $d G_{i}+\left(\left(\Pi, G_{i}\right)\right)+\iota_{i} \Pi=D_{i}$

The Soloviev bracket $\left(\left(G_{i}, G_{j}\right)\right)$ is the symmetrization of the Soloviev bracket

$$
\left(\left(\frac{1}{2} \epsilon_{i k \ell}\left\langle A^{+k}, A^{+\ell}\right\rangle,\left\langle A_{j}, c^{+}\right\rangle\right)\right)=-\epsilon_{i j k}\left\langle A^{+k}, c^{+}\right\rangle
$$

in the indices $i$ and $j$, hence it vanishes. This completes the proof of the following result.

Proposition 4.5. The abelian Chern-Simons theory $\Pi+u^{i} G_{i}$ is a covariant field theory.

Let $\Gamma=\Gamma_{1} \Gamma_{2} \Gamma_{3}$. The operation $\Gamma: \tau_{\geq 1} C^{*+3}(\mathfrak{g}) \rightarrow\left(\mathbb{F}^{*}, d+\pi\right)$ induces a quasiisomorphism of complexes. Similarly, the operation

$$
f(c) \in C^{k}(\mathfrak{g}) \mapsto \int \Gamma f(c)=\int\left(\int G_{1},\left(\int G_{2},\left(\int G_{3}, \int f(c)\right)\right)\right) \in \mathcal{F}^{k-3}
$$

induces a quasi-isomorphism of complexes from $\tau_{\geq 1} C^{*+3}(\mathfrak{g})$ to $\left(\mathcal{F}^{*}, \pi\right)$. Introduce the operations

$$
\mathcal{D}\left(A_{i}\right)=\operatorname{pr}\left\langle A_{i}, \frac{\partial}{\partial c}\right\rangle+\frac{1}{2} \sigma_{i}(\mathrm{E}-2), \quad \mathcal{D}\left(A^{+i}\right)=\operatorname{pr}\left\langle A^{+i}, \frac{\partial}{\partial c}\right\rangle, \quad \mathcal{D}\left(c^{+}\right)=\operatorname{pr}\left\langle c^{+}, \frac{\partial}{\partial c}\right\rangle
$$

on $\mathbb{F}$. For $f(c) \in C^{k}(\mathfrak{g}) \subset \mathbb{F}^{0, k}$, we have the formula

$$
\begin{aligned}
\Gamma f(c) & =-\mathcal{D}\left(A_{1}\right) \mathcal{D}\left(A_{2}\right) \mathcal{D}\left(A_{3}\right) f(c)+\mathcal{D}\left(A_{i}\right) \mathcal{D}\left(A^{+i}\right) f(c)-\mathcal{D}\left(c^{+}\right) f(c) \\
& \in \mathbb{F}^{k-3}
\end{aligned}
$$

Given a pair $f(c), g(c) \in C^{*}(\mathfrak{g})$ of Lie algebra cochains, the bracket $((\Gamma f(c), g(c)))$ is given by the formula

$$
\{f, g\}=\left\langle\frac{\partial f(c)}{\partial c}, \frac{\partial g(c)}{\partial c}\right\rangle .
$$

In the next section, we extend the results of this section to when $\mathfrak{g}$ is non-abelian. 


\section{The covariant non-abelian Chern-Simons action}

In this section, we construct the covariant extension of the classical field theory of non-abelian Chern-Simons theory, following the approach of section 3.

Suppose that the inner product space $\mathfrak{g}$ considered in the last section underlies a real Lie algebra with bracket $[-,-]$ and invariant inner product $\langle-,-\rangle$. (We do not assume that the inner product is definite, only that it is nondegenerate.) We consider the local functional

$$
\rho=\frac{1}{6}\langle c,[c, c]\rangle .
$$

This is certainly a cocycle for the abelian Chern-Simons theory of the previous section, of ghost number 3 .

By (4.1), we see that

$$
\begin{aligned}
\Gamma \rho= & \frac{1}{6} \epsilon^{i j k}\left\langle A_{i},\left[A_{j}, A_{k}\right]\right\rangle-\frac{1}{2}\left\langle c^{+},[c, c]\right\rangle-\left\langle A^{+i},\left[A_{i}, c\right]\right\rangle \\
& +\frac{1}{4} \sigma_{i}\left(\left\langle A^{+i},[c, c]\right\rangle+\epsilon^{i j k}\left\langle\left[A_{j}, A_{k}\right], c\right\rangle\right) \\
& -\frac{1}{16} \sigma_{i} \sigma_{j} \epsilon^{i j k}\left\langle A_{k},[c, c]\right\rangle-\frac{1}{288} \sigma_{i} \sigma_{j} \sigma_{k} \epsilon^{i j k}\langle c,[c, c]\rangle .
\end{aligned}
$$

By (4.2), we see that

$$
((\rho, \Gamma \rho))=-\frac{1}{4}\langle[c, c],[c, c]\rangle=-\frac{1}{4}\langle c,[c,[c, c]]\rangle=0,
$$

since $[c,[c, c]]$ vanishes by the Jacobi relation. In this way, we obtain the following explicit solution of the covariant classical master equation for Chern-Simons theory:

$$
\begin{aligned}
S_{u}= & \Pi+\Gamma \rho+u^{i} G_{i} \\
= & \operatorname{cs}_{3}(\mathbb{A})-\frac{1}{4} \sigma_{k}\left(\left\langle A^{+k},[c, c]\right\rangle+\epsilon^{i j k}\left\langle c,\left[A_{i}, A_{j}\right]\right\rangle\right) \\
& -\frac{1}{16} \sigma_{j} \sigma_{k} \epsilon^{i j k}\left\langle A_{i},[c, c]\right\rangle+\frac{1}{288} \sigma_{i} \sigma_{j} \sigma_{k} \epsilon^{i j k}\langle c,[c, c]\rangle+u^{i} G_{i} .
\end{aligned}
$$

We conclude that the morphism $\Gamma: \tau_{\geq 1} C^{*+k}(\mathfrak{g}) \rightarrow \mathbb{F}^{*}$ of (4.1) works as well in the non-abelian case, giving rise to a quasi-isomorphism of complexes of degree -3 from the reduced Lie algebra cochains $\left(\tau_{\geq 1} C^{*}(\mathfrak{g}), \delta\right)$ of the Lie algebra $\mathfrak{g}$ to the Batalin-Vilkovisky complex $(\mathbb{F}, d+\mathrm{s})$ of classical Chern-Simons theory:

$$
H^{k}(\mathcal{F}, \mathbf{s}) \cong \begin{cases}H^{k+3}(\mathfrak{g}), & k \geq-2, \\ 0, & k<-2 .\end{cases}
$$

If $\mathfrak{g}$ is semisimple, Whitehead's lemma shows that $H^{1}(\mathfrak{g}) \cong H^{2}(\mathfrak{g}) \cong 0$, and hence that $H^{*}(\mathcal{F}, \mathrm{s})$ vanishes in negative degree. These results were obtained Barnich et al. [2, section 14].

Barnich and Grigoriev [3] have given a geometric interpretation of this result. (Their results more generally for any AKSZ field theory: Chern-Simons theory was the original example that led to the introduction of the class of AKSZ field theories.) If $\mathfrak{g}$ is a reductive Lie algebra over $\mathbb{R}$ with invariant inner product $\langle-,-\rangle$, the graded manifold $\mathfrak{g}[1]$ has the 
graded commutative algebra of Lie algebra cochains $C^{*}(\mathfrak{g})$ as its ring of functions. Denote the coordinate functions by $c \in C^{1}(\mathfrak{g}) \otimes \mathfrak{g}$. The inner product determines a symplectic form on $\mathfrak{g}[1]$,

$$
\Omega=\frac{1}{2}\langle d c, d c\rangle,
$$

with associated Poisson bracket

$$
\{f, g\}=(-1)^{|f|}\left\langle\frac{\partial f}{\partial c}, \frac{\partial g}{\partial c}\right\rangle .
$$

The differential $\delta$ on the complex of Lie algebra cochains may be interpreted geometrically as the Hamiltonian vector field associated to the cochain $\rho$. In this setting, Barnich and Grigoriev prove the existence of a quasi-isomorphism

$$
\left(\tau_{\geq 1} C^{*+3}(\mathfrak{g}), \delta\right) \rightarrow(\mathcal{F}, \mathbf{s}) .
$$

In this section, we have obtained a lift of this quasi-isomorphism of complexes to a quasiisomorphism of shifted differential graded Lie algebras.

In the appendix, we prove the purely algebraic result that the differential graded $(-2)$ shifted Lie algebra $C^{*}(\mathfrak{g})$ associated to a reductive Lie algebra $\mathfrak{g}$ with invariant inner product $\langle-,-\rangle$ is homotopy abelian. This implies the following result for the sheaf of 1-shifted differential graded Lie algebras $\mathcal{F}$ on $\mathbb{R}^{3}$ associated to the corresponding Chern-Simons theory.

Theorem 5.1. The Batalin-Vilkovisky complex $(\mathcal{F}, \mathrm{s})$ associated to the Chern-Simons theory of a semisimple Lie algebra is homotopy abelian, and there is a quasi-isomorphism of differential graded 1-shifted Lie algebras $\left(\tau_{\geq 1} H^{*}(\mathfrak{g}), 0\right) \hookrightarrow(\mathcal{F}, \mathrm{s})$.

It follows that the deformation theory of $(\mathcal{F}, s)$ is unobstructed: the moduli space of deformations is naturally isomorphic to the space of invariant inner products on $\mathfrak{g}$. Note that the first obstruction is identically zero, since $H^{4}(\mathfrak{g})=0$ for a semisimple Lie algebra $\mathfrak{g}$.

\section{A The Chevalley-Eilenberg complex of a semisimple Lie algebra}

If $\mathfrak{g}$ is a reductive Lie algebra, Chevalley and Eilenberg prove that the inclusion

$$
C^{*}(\mathfrak{g})^{\mathfrak{g}} \subset C^{*}(\mathfrak{g})
$$

of invariant cochains is a quasi-isomorphism. Since the differential vanishes on $C^{*}(\mathfrak{g})^{\mathfrak{g}}$, there is a natural isomorphism $H^{*}(\mathfrak{g}) \cong C^{*}(\mathfrak{g})^{\mathfrak{g}}$.

An invariant inner product $\langle-,-\rangle$ on $\mathfrak{g}$ induces a $(-2)$-shifted Poisson bracket on $C^{*}(\mathfrak{g})$, making $C^{*}(\mathfrak{g})$ into a differential graded $(-2)$-shifted Lie algebra. Let $\theta \in C^{1}(\mathfrak{g}, \mathfrak{g})$ be the 1-cochain on $\mathfrak{g}$ given by the identity map; think of $\theta$ as a coordinate system on the graded manifold $\mathfrak{g}[1]$, and cochains as functions on $\mathfrak{g}[1]$. The Poisson bracket of $f \in C^{k}(\mathfrak{g})$ and $C^{\ell}(\mathfrak{g})$ equals

$$
\{f, g\}=(-1)^{k}\left\langle\frac{\partial f}{\partial \theta}, \frac{\partial g}{\partial \theta}\right\rangle .
$$

The differential $\delta$ of $C^{*}(\mathfrak{g})$ equals $\operatorname{ad}(\rho)$, where is $\rho$ is the invariant 3 -cochain $\frac{1}{6}\langle\theta,[\theta, \theta]\rangle$. In particular, $\delta \theta=-\frac{1}{2}[\theta, \theta]$. Let $\Theta=\frac{1}{2}[\theta, \theta]$. 
The purpose of this appendix is to record a proof of the following result.

Proposition A.1. If $\mathfrak{g}$ is semisimple and $f$ and $g$ are invariant cochains, $\{f, g\}=0$.

This result implies that the differential graded Lie algebra $C^{*}(\mathfrak{g})$ is formal, and that the differential graded Lie algebra $H^{*}(\mathfrak{g})$ is abelian. It follows that the same statement holds for the sheaf of differential graded Lie algebras $(\mathcal{F}, s)$ for the Chern-Simons theory with semisimple Lie algebra $\mathfrak{g}$.

Let $S(\mathfrak{g})$ be the space of polynomials on the finite-dimensional Lie algebra $\mathfrak{g}$ with invariant inner product $\langle-,-\rangle$. The gradient $\nabla P \in S(\mathfrak{g}) \otimes \mathfrak{g}$ of a polynomial $P(x)$ is given by the formula

$$
\langle\nabla P(x), y\rangle=\left.\frac{d}{d s}\right|_{s=0} P(x+s y) .
$$

Let $I(\mathfrak{g})$ be the subspace of invariant polynomials: $P \in I(\mathfrak{g})$ is invariant if and only if for all $x, y \in \mathfrak{g}$,

$$
\langle\nabla P(x),[y, x]\rangle=0 .
$$

Grade the algebra $I(\mathfrak{g})$ by placing polynomials homogeneous of degree $\ell$ in degree $2 \ell$, making it into a graded commutative algebra, and let $I_{+}(\mathfrak{g})$ be the subspace of invariant polynomials with vanishing constant term.

Define a map

$$
\tau: I(\mathfrak{g}) \rightarrow C^{*}(\mathfrak{g})[-1]
$$

by the formula

$$
\tau P=(2 \ell-1)^{-1}\langle\nabla P(\Theta), \theta\rangle .
$$

As an example, conside the quadratic form $Q(x)=\frac{1}{2}\langle x, x\rangle$ associated to the invariant bilinear form $\langle x, y\rangle$ on $\mathfrak{g}$, gives rise to the cocycle $\tau Q=\rho$.

Lemma A.2. Let $\mathfrak{g}$ be a reductive Lie algebra. Let $P \in I(\mathfrak{g})$. Then

1) $P(\Theta)=0$, and

2) $\partial(\tau P) / \partial \theta=\nabla P(\Theta)$.

Proof. Since $\mathfrak{g}$ is reductive, $I(\mathfrak{g})$ is spanned by trace polynomials of the form

$$
P_{V, \ell}(x)=\operatorname{Tr}_{V}\left(\rho(x)^{\ell}\right),
$$

where $\rho: \mathfrak{g} \rightarrow \operatorname{gl}(V)$ is a finite-dimensional representation of $\mathfrak{g}$ and $\ell>0$. (See Humphreys [9, section 23.1]; this is a theorem of Chevalley, which follows from the Weyl character formula.) But $\rho(\Theta)=\frac{1}{2}[\rho(\theta), \rho(\theta)]=\rho(\theta)^{2}$, so

$$
P_{V, \ell}(\Theta)=\operatorname{Tr}_{V}\left(\rho(\theta)^{2 \ell}\right)=\frac{1}{2} \operatorname{Tr}_{V}\left[\rho(\theta), \rho(\theta)^{2 \ell-1}\right]=0 .
$$

It follows that

$$
\tau P_{V, \ell}=\frac{\ell}{2 \ell-1} \operatorname{Tr}_{V}\left(\rho(\Theta)^{\ell-1} \rho(\theta)\right)=\frac{\ell}{2 \ell-1} \operatorname{Tr}_{V}\left(\rho(\theta)^{2 \ell-1}\right),
$$

and

$$
\frac{\partial\left(\tau P_{V, \ell}\right)}{\partial \theta}=\ell \rho(\Theta)^{\ell-1}
$$


Corollary A.3. If $\mathfrak{g}$ is reductive, $\tau$ maps $I^{2 \ell}(\mathfrak{g})$ to the space of cocycles $Z^{2 \ell-1}(\mathfrak{g}) \subset C^{2 \ell-1}(\mathfrak{g})$, and the restriction of $\tau$ to $I^{+}(\mathfrak{g})^{2}$ vanishes.

Proof. Let $P$ be a homogeneous invariant polynomial of degree $\ell>0$. Since $d \theta=\Theta$, we have

$$
(2 \ell-1) d(\tau P)=\langle\nabla P(\Theta), \Theta\rangle=\ell P(\Theta),
$$

which vanishes by the lemma.

Consider $P, Q \in I_{+}(\mathfrak{g})$, of degree $\ell$ and $m$ respectively. The map $T$ vanishes on $P Q$ :

$$
(2 \ell+2 m-1) \tau(P Q)=\langle\nabla P(\Theta), \theta\rangle Q(\Theta)+P(\Theta)\langle\nabla Q(\Theta), \theta\rangle=0 .
$$

We may choose a sequence $P_{i} \in I^{2 \ell_{i}}(\mathfrak{g}), 1 \leq i \leq r$, of homogeneous invariant polynomials whose images form a basis of $I_{+}(\mathfrak{g}) / I_{+}(\mathfrak{g})^{2}$. The numbers $\left(\ell_{1}, \ldots, \ell_{r}\right)$ are called the exponents of $\mathfrak{g}$, and the natural number $r$ is the rank of $\mathfrak{g}$. Chevalley proves that $I(\mathfrak{g})$ is a polynomial algebra with generators $P_{i}$. The map $\tau: I(\mathfrak{g}) \rightarrow C^{*}(\mathfrak{g})[-1]$ induces a morphism from $I_{+}(\mathfrak{g}) / I_{+}(\mathfrak{g})^{2}$ to $Z^{*}(\mathfrak{g})[-1]$. Borel proves that $H^{*}(\mathfrak{g})$ is an exterior algebra generated by $\left[\tau P_{i}\right] \in H^{2 \ell_{i}-1}(\mathfrak{g})$.

For the classical groups, the generators $P_{i} \in I(\mathfrak{g})$ are trace polynomials associated to the fundamental representation, with a single exception, the $\operatorname{Pfaffian} \operatorname{Pf}(x) \in I(\mathbf{s o}(2 m))$, which is the difference of two trace polynomials associated to the two half-spinor representations $\rho_{ \pm}: \mathbf{s o}(2 m) \rightarrow S_{ \pm}:$

$$
\operatorname{Pf}(x)=i^{n}\left(\operatorname{Tr}_{S_{+}}\left(\rho_{+}(x)^{m}\right)-\operatorname{Tr}_{S_{-}}\left(\rho_{-}(x)^{m}\right)\right) .
$$

Chevalley's result is only really needed in the proof of Lemma A.2 to handle the possible presence of the exceptional Lie algebras.

It follows that the inclusion of the subalgebra of $C^{*}(\mathfrak{g})$ spanned by the cocycles

$$
\tau P_{i_{1}} \ldots \tau P_{i_{k}} \in Z^{2 \ell_{i_{1}}+\cdots+2 \ell_{i_{k}}-k}(\mathfrak{g}), \quad 1 \leq i_{1}<\cdots<i_{k} \leq r,
$$

induces a quasi-isomorphism of dg commutative algebras $H^{*}(\mathfrak{g}) \hookrightarrow C^{*}(\mathfrak{g})$. The image of this morphism is the subalgebra $C^{*}(\mathfrak{g})^{\mathfrak{g}}$ of invariant cochains.

Proposition A.1 is now a consequence of the following lemma.

Lemma A.4. If $P \in I(\mathfrak{g})$ and $Q \in I(\mathfrak{g})$ are homogeneous of degree $\ell, m>1$,

$$
\{\tau P, \tau Q\}=0 .
$$

Proof. By Lemma A.2

$$
\{\tau P, \tau Q\}=\left\langle\frac{\partial(\tau P)}{\partial \theta}, \frac{\partial(\tau Q)}{\partial \theta}\right\rangle=\langle\nabla P(\Theta), \nabla Q(\Theta)\rangle .
$$

Since $\langle\nabla P, \nabla Q\rangle$ is invariant and homogeneous of degree $\ell+m-2>0$, Lemma A.2 implies the vanishing of $\langle\nabla P(\Theta), \nabla Q(\Theta)\rangle$.

Open Access. This article is distributed under the terms of the Creative Commons Attribution License (CC-BY 4.0), which permits any use, distribution and reproduction in any medium, provided the original author(s) and source are credited. 


\section{References}

[1] S. Axelrod and I.M. Singer, Chern-Simons perturbation theory, in International Conference on Differential Geometric Methods in Theoretical Physics, pp. 3-45 (1991) [hep-th/9110056] [INSPIRE].

[2] G. Barnich, F. Brandt and M. Henneaux, Local BRST cohomology in gauge theories, Phys. Rept. 338 (2000) 439 [hep-th/0002245] [INSPIRE].

[3] G. Barnich and M. Grigoriev, A Poincaré lemma for sigma models of AKSZ type, J. Geom. Phys. 61 (2011) 663 [arXiv:0905.0547] [INSPIRE].

[4] A.S. Cattaneo and G. Felder, On the AKSZ formulation of the Poisson sigma model, Lett. Math. Phys. 56 (2001) 163 [math/0102108] [INSPIRE].

[5] A.S. Cattaneo, P. Mnev and N. Reshetikhin, Classical BV theories on manifolds with boundary, Commun. Math. Phys. 332 (2014) 535 [arXiv:1201.0290] [inSPIRE].

[6] E. Getzler, A Darboux theorem for Hamiltonian operators in the formal calculus of variations, Duke Math. J. 111 (2002) 535 [math/0002164].

[7] E. Getzler, Covariance in the Batalin-Vilkovisky formalism and the Maurer-Cartan equation for curved Lie algebras, Lett. Math. Phys. 109 (2019) 187 [arXiv:1801.04525] [INSPIRE].

[8] W.M. Goldman and J.J. Millson, The deformation theory of representations of fundamental groups of compact Kähler manifolds, Inst. Hautes Études Sci. Publ. Math. 67 (1988) 43.

[9] J.E. Humphreys, Introduction to Lie algebras and representation theory, Graduate Texts in Mathematics, Vol. 9, Springer-Verlag, New York-Berlin (1972), pp. xii+169 [DOI].

[10] P.J. Olver, Applications of Lie groups to differential equations, Graduate Texts in Mathematics, Vol. 107, Springer-Verlag, New York (1986), pp. xxvi+497 [DOI].

[11] V.O. Soloviev, Boundary values as Hamiltonian variables. 1. New Poisson brackets, J. Math. Phys. 34 (1993) 5747 [hep-th/9305133] [INSPIRE].

[12] D. Sullivan, Infinitesimal computations in topology, Inst. Hautes Études Sci. Publ. Math. 47 (1977) 269. 